\title{
ETUDE SUR MODELE DU COMPORTEMENT DES OUVRAGES D'ACCOSTAGE SOUMIS A DES SOLLICITATIONS DYNAMIQUES
}

\author{
Abdellah Alem ; Jean-Georges Sieffer \\ Chercheur au laboratoire de Génie Civil, E.N.S.M, Nantes; \\ Professeur, Directeur du laborawire de Génie Civil, E.N.S.M, Nantes.
}

\begin{abstract}
The failure of certain number of structures show the weakness of static calculation methods which do not take into account the dynamic loads of the berthing ships. Trying to understand how it works, a comparative study was carried out in the laboratory using the plane model of Schneebeli. Static tests had been done. The subgrade reaction peculiar to the model was obtained by comparing and correlating the field data and the response predicted using the program Pilate developed in the Laboratoire Central des Ponts et Chaussées.

Dynamic tests were conducted using an impact of a mass to simulate the induced forces of ships on berthing structures. The effects of the impact mass, the impact velocity, the embedded length and the plate rigidity were studied.

In other respect, a numerical method was developed. A good agreement between the measured response and the predicted one was found.
\end{abstract}

\section{RESUME}

Les désordres se produisant dans certains ouvrages d'accostages, en particulier au niveau de l'encastrement dans le sol des ducs d'albe, mettent en évidence les insuffisances des méthodes de calcul statiques qui ne prennent pas en compte les sollicitations dynamiques appliquées par les navires au moment de l'accostage.

De façon à mieux comprendre les phénomènes mis en jeu, une étude expérimentale comparative a été entreprise en laboratoire sur un modèle plan de Schneebeli. Des essais systématiques ont été réalisés en statique en faisant varier la fiche et la rigidité de la plaque. Le module de réaction propre au modele a été obtenu par calage des résultats expérimentaux à l'aide du programme Pilate développé par le Laboratoire Central des Ponts et Chaussées.

Les essais dynamiques ont été menés en simulant les sollicitations dues aux navires par des impacts provoqués par une masse. Les influences de la fiche et de la rigidité de la plaque, de la masse d'impact et de la vitesse d'impact ont été analysées.

Par ailleurs, une solution numérique a pu être développée. Une bonne corrélation entre la réponse prédite et mesurée est obtenue. 


\section{DISPOSITIF EXPERIMENTAL}

Le dispositif expérimental utilisé est représenté par la figure 1.

\subsection{Matériau du modèle de Schneebeli}

Nous avons utilisé des rouleaux en duralumininium. Ces rouleaux sont de deux diamètres ( 3 et $5 \mathrm{~mm}$ ) également répartis, leur longeur est de $6 \mathrm{~cm}$. La densité du matériau en place est de 2.29 et l'angle de frottement interne de $27^{\circ}$.

\subsection{Plaques}

Les plaques sont en acier. Elles sont de 1 mètre de longueur et de $8 \mathrm{~cm}$ de largeur, soit $2 \mathrm{~cm}$ de plus que la longueur des rouleaux, ce qui permet la mise en place des dispositifs de mesure à l'extérieur du modèle.

Dans le but d'étudier l'influence de la rigidité de la plaque, quatre épaisseurs ont été utilisées (ep $=2.5 \mathrm{~mm}, 4.0 \mathrm{~mm}, 6.0 \mathrm{~mm}, 8.0 \mathrm{~mm}$ ).

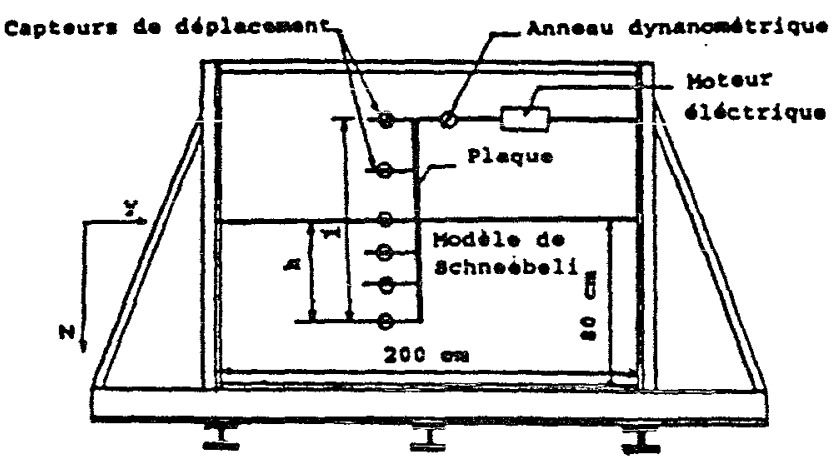

Fig. 1 - Dispọsitif expérimental utilisé

\section{DETERMINATION DES COURBES DE REACTION DU "SOL"}

Les courbes sont obtenues à partir des essais statiques. La plaque est sollicitée en flexion. L'effort en tête ainsi que les déformations de la plaque sont enregistrées.

Les paramètres définissant les courbes $\left(\mathrm{P}_{\mathrm{st}}-\mathrm{y}\right)$ de la figure 2 , sont définis par calage à l'aide du programme Pilate élaboré au Laboratoire Central des Ponts et Chaussées. Ces paramètres sont indépendants de la fiche et de la rigidité de la plaque. Une bonne corrélation entre les résultats expérimentaux et 
la réponse prédite est obtenue comme le montre la figure 2 donnée à titre d'exemple.

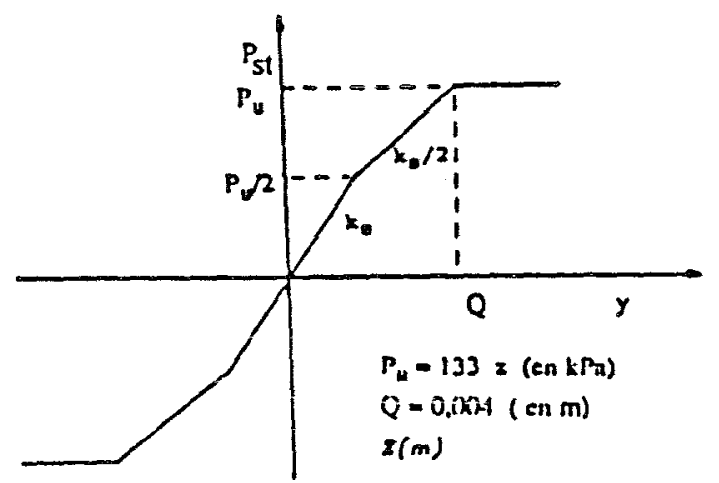

Fig. 2 - Courbe de réaction du "sol"

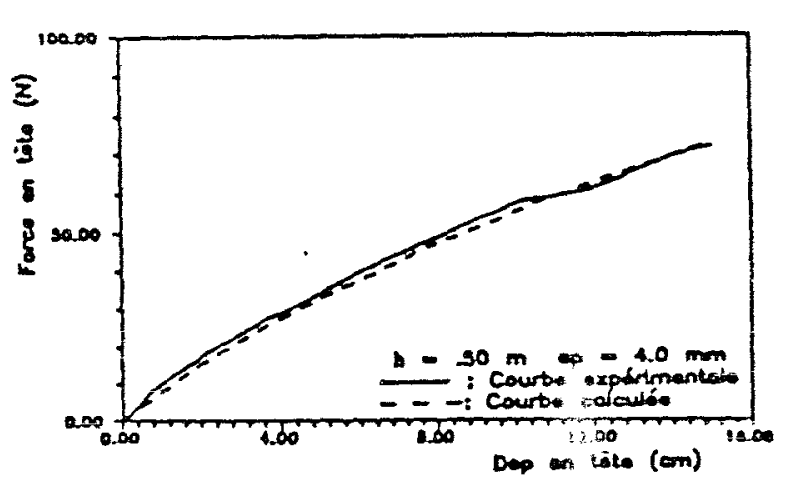

Fig. 3 - Force en tête en tonction du déplacement en tête

\section{RESULTATS EXPERIMENTAUX DES ESSAIS EN DYNA- MIQUE}

Le même dispositif expérimental que celui utilisé dans l'étude statique est utilisé. La mise en charge de la plaque est réalisée par l'impact en tête d'une masse M.

La base du dispositif de mesure est constituée d'un accéléromètre et d'un capteur de force fixés en tête de la plaque et reliés à un système d'acquisition composé essentiellement d'un oscilloscope numérique et d'un calculateur.

On enregistre l'accélération et la force en tête de la plaque soumise à l'impact d'une masse $\mathrm{M}$. La vitesse et le déplacement en tête sont déduits de l'accélération par intégrations. Les figures (4.1, 4.2, 4.3 et 4.4$)$ montrent un exemple de résultats d'un essai, avec:

masse d'impact : $M=5 \mathrm{~kg}$,

vitesse d'impact: $V=1.09 \mathrm{~m} / \mathrm{s}$,

épaisseur de la plaque : $\mathrm{ep}=4.0 \mathrm{~mm}$,

fiche $: h=0.5 \mathrm{~m}$.

Fig. 4.1 - Force en tête en fonction du temps
Fig. 4.2 - Accélération en tête en fonction du temps
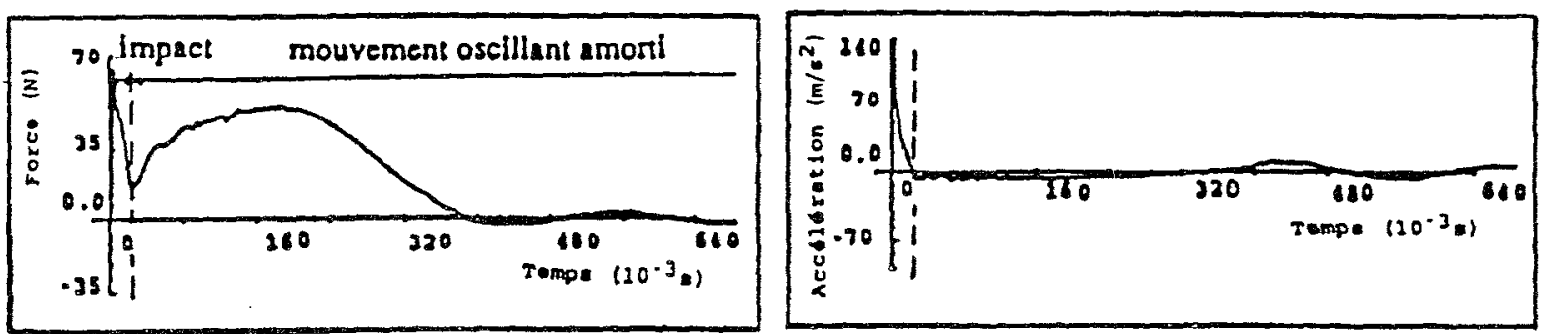


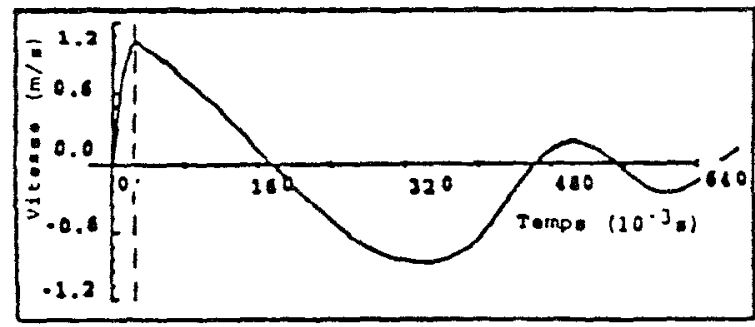

Figure 4.3 - Vitesse en tête en fonction du temps

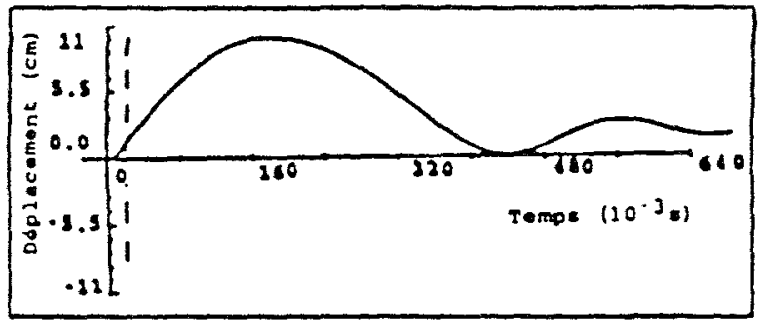

Figure 4.4 - Déplacement en téte en fonction du temps

Les courbes représentatives de la force et de l'accélération en tête, figures (4.1 et 4.2) font apparaître deux phases.

a) La phase d'impact : La force et l'accélération augmentent d'abord assez rapidement jusqu'à un maximum puis diminuent également rapidement. Cette phase correspond à la mise en vitesse de la plaque. La fin de cette phase est caractérisée par le changement de signe de l'accélération, ce qui correspond au minimum de la force en tête de la plaque et au maximum de la vitesse en tête .

b) La deuxième phase : Elle correspond à un mouvement oscillant amorti de la plaque, comme le montrent bien les courbes de vitesse et de déplacement, figures ( 4.3 et 4.4 ).

\section{MODELISATION ET ALGORITHME DE CALCUL :}

Un algorithme de calcul est développé pour prédire le comportement des plaques soumises à l'impact d'une masse $\mathrm{M}$. Dans un premier temps, on a cherché à modéliser la masse d'impact et le comportement non linéaire du "sol".

\subsection{Modélisation de la masse d'impact :}

La simulation des corps déformables, lors des problèmes de choc, fait généralement appel aux modèles utilisés dans la théorie des vibrations des systèmes discrets. Ces modèles sont souvent une combinaison de modèles à un élément, possédant une propriété quelconque (élasticité - viscosité plasticité ). La modélisation retenue de la masse pendant la phase d'impact est représentée par la figure 5.a. Il s'agit d'une masse attachée à un système ressortamortisseur. Les valeurs de la raideur $\mathrm{K}$ et de l'amortisseur $\mathrm{C}$ sont obtenues par ajustement avec des résultats expérimentaux . 
Pendant la deuxième phase, on considère que la masse d'impact reste collé à la plaque.

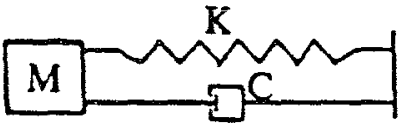

5.a. Phase dimpact

\section{$\mathrm{M}$}

$5 . b$ - Phase doscillations amorties

Fig. 5 - Schéma de modélisation de la masse ơmpact

\subsection{Modélisation du comportement du "sol" :}

La description analytique du comportement non-linéaire du sol a toujours été un problème génant pour l'ingénieur. Une apprọche généralemenent acceptée est la simulation du comportement du sol par un modèle rhéologique. Ainsi, SMITH [9], SAMSON, HIRSH et LOWERY [8] introduisirent le modèle de KELVIN VOIGT modifié dans l'analyse du battage des pieux.

ROSS [7] développa une solution analytique pour la réponse en dynamique des pieux en offshore soumis à des chargements latéraux. Il utilisa le modèle de KELVIN VOIGT de SMITH modifié pour la modélisation du compontement non-linéaire du sol.

Notre idée est d'utiliser une modélisation semblable à celle suggérée par ROSS. Ainsi, on a choisi de conserver la modélisation du sol en statique et d'introduire l'effet de la vitesse de chargement s'il y a lieu.

Le diagramme schématique du modèle retenu, avec ses caractéristiques, est représenté sur la figure 6.

Fig. 6 - Modèle méologique avec ses caractéristiques
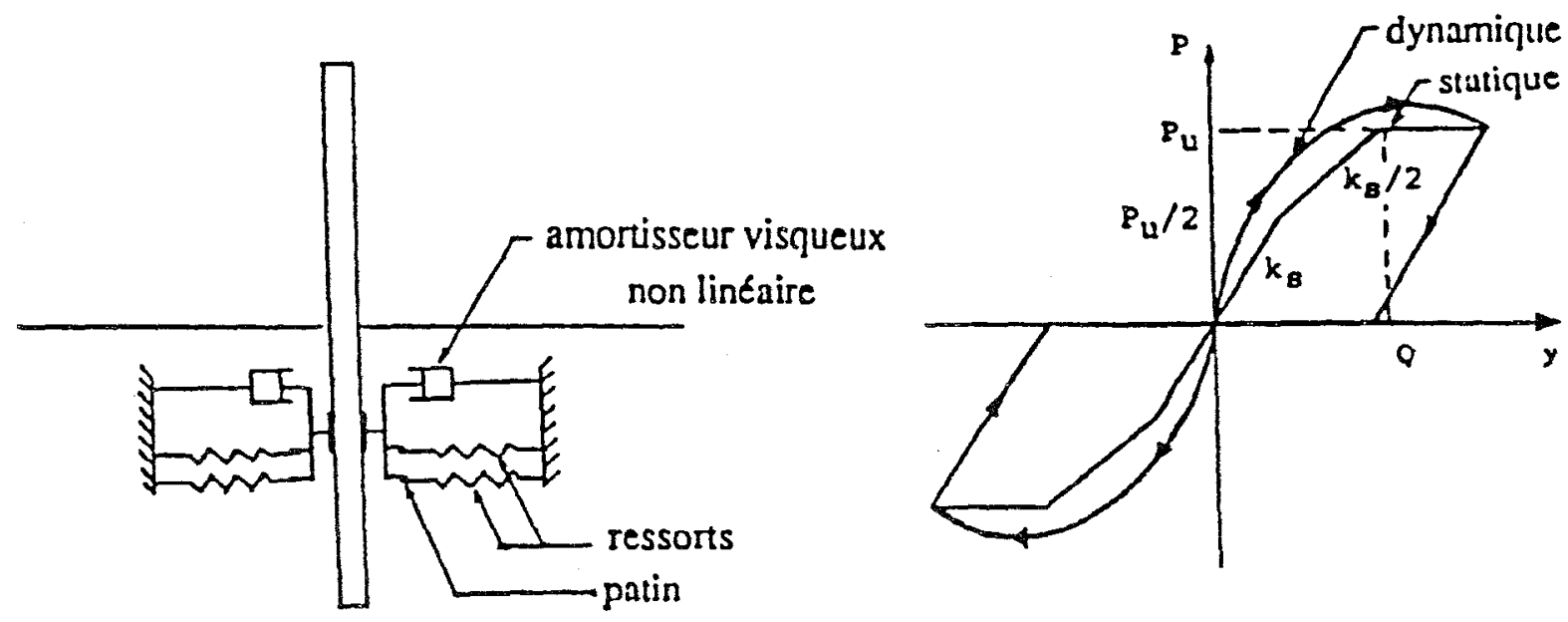
Le modèle est exprimé en équation comme suit :

$$
\begin{aligned}
& \mathrm{P}_{\mathrm{dyn}}=\mathrm{P}_{\mathrm{st}}\left(1+\mathrm{J}|\mathrm{V}|^{\mathrm{N}}\right) \\
& \text { avec } \quad \mathrm{P}_{\mathrm{dyn}} \text { : chargement dynamique, } \\
& \mathrm{P}_{\mathrm{st}} \text { : chargement statique, } \\
& \mathrm{J}: \text { facteur d'amortissement du " sol ". } \\
& \mathrm{V}: \text { vitesse de chargement, } \\
& \mathrm{N} \text { : exposant de la vitesse. }
\end{aligned}
$$

Le facteur d'amortissement $\mathrm{J}$ dans notre expérimentacion est trouvé égal à 0 .

\subsection{Présentation de l'algorithme :}

La plaque est discrétisée en éléments poutres. Les conditions d'équilibre dynamique au niveau de chaque noeud de chaque élément permettent d'aboutir au système dynamique non-linéaire qui se formule de la manière suivante :

$[M]\{\Gamma\}+[C]\{V\}+[K]\{U\}=\{F\}$

avec:

$\{U\},\{V\},\{\Gamma\}$ sont respectivement les vecteurs des déplacements, des vitesses et des accélérations aux nœuds.

[ $M$ ] est la matrice de masse obtenue par assemblage des matrices élémentaires $[\mathrm{M}]_{\mathrm{e}}$ calculées par la relation :

$$
[M]_{e}=\int_{0}^{1} \rho S[A(x)]^{T}[A(x)] d x
$$

où $\quad \rho$ est la masse volumique $\mathrm{du}$ " sol ",

$S$ est la section de la plaque,

1 est la longueur de l'élément poutre.

$[A(x)]=\left[N_{1}(x), N_{2}(x), N_{3}(x), N_{4}(x)\right]$ 
$N_{1}(x)=1-3\left(\frac{x}{1}\right)^{2}+2\left(\frac{x}{1}\right)^{3}$

$N_{2}(x)=1\left(\frac{x}{l}-2\left(\frac{x}{l}\right)^{2}+\left(\frac{x}{1}\right)^{3}\right)$

$N_{3}(x)=3\left(\frac{x}{l}\right)^{2}-2\left(\frac{x}{l}\right)^{3}$

$N_{4}(x)=-1\left(\left(\frac{x}{I}\right)^{2}-\left(\frac{x}{I}\right)^{3}\right)$

[ $\mathrm{K}$ ] est la matrice de rigidité obtenue par assemblage des matrices de rigidité élémentaires $[\mathrm{K}]_{\mathrm{e}}$ calculées par la relation :

$[K]_{e}=E I \int_{0}^{1}\left[\frac{\partial^{2} A(x)}{\partial x^{2}}\right]^{T}\left[\frac{\partial^{2} A(x)}{\partial x^{2}}\right] d x$

dans laquelle $\mathrm{E} I$ est la rigidité de la plaque.

[C] est la matrice d'amortissement.

\{ $F$ \} est le vecteur des forces nodales, dépendant des déformations du "sol" et obtenu par assemblage des vecteurs des forces élémentaires $\{\mathrm{F}\}_{\mathrm{e}}$ calculés par la relation :

$[F\}_{\mathrm{e}}=\int_{0}^{1}[A(x)]^{T} P(x) d x$

où $\mathrm{P}(\mathrm{x})$ sont les forces de pression du " sol ".

- Un réassemblage des matrices de masse, de rigidité et du vecteur de forces nodales est nécessaire, pendant la phase d'impact, pour prendre en compte l'équation de mouvement supplémentaire de la masse d'impact $M$ et des forces d'impact sur la plaque.

- De la même manière, pendant la phase d'oscillations amorties, une correction de la matrice de masse est nécessaire pour prendre en compte la masse d'impact considérée collée à la plaque.

- Le système ( 2 ) est résolu par une méthode de calcul pas à pas. En utilisant les différences retardées pour approcher $\mathrm{V}$ et $\Gamma$ : 


$$
\begin{aligned}
& V_{t}=\frac{U_{t}-U_{t-\Delta t}}{\Delta t} \\
& \Gamma_{t}=\frac{V_{t}-V_{t-\Delta t}}{\Delta t}
\end{aligned}
$$

où $\Delta t$ est le pas de temps choisi,

nous résolvons à chaque étape le système suivant :

$\left[[M]+[K] \Delta t^{2}+[C] \Delta t\right]\left\{U_{t}\right\}=[[M]+[C] \Delta t]\left(U_{t-\Delta t}\right\}+[M]\left\{V_{t-\Delta t}\right\}+\left\{F_{t}\right\} \Delta t^{2}$

On passe de la phase d'impact à la phase d'oscillations amorties lorsque la condition suivante est réalisée.

$\mathrm{V}_{0 \mathrm{t}} \leq \mathrm{V}_{1 \mathrm{t}}$

où $\quad V_{0 t}$ est la vitesse de la masse d'impact au temps $t$, $\mathrm{V}_{1 \mathrm{t}}$ est la vitesse de déplacement de la plaque au point où il y a impact.

Les figures (7.1, 7.2, 7.3 et 7.4) présentent, pour un essai, une comparaison des résultats expérimentaux avec les résultats de calcul par cet algorithme.

Les figures $(8.1,8.2,8.3$ et 8.4$)$ donnent une comparaison des déplacements maximaux expérimentaux et calculés, pour les autres essais.

D'une manière générale, une bonne corrélation entre les résultats expérimentaux et la réponse prédite est obtenue. La corrélation est d'autant meilleure que la fiche augmente.

\section{CONCLUSION :}

On propose un algorithme pour prédire le comportement du modèle simulant l'ouvrage d'accostage et soumis à l'impact d'une masse $\mathrm{M}$ en tête.

Une bonne corrélation entre la réponse prédite et mesurée est obtenue.

L'algorithme développé peut servir pour prédire le comportement des ouvrages d'accostage soumis aux impacts de navires accostant ou amarrant. 


\section{REFERENCES :}

[ 1 ] Bangratz. J. G, Frank . R, et Romagny M. 1981. Programme de calcul d'un pieu isolé soumis d des efforts de flexion en tête et d des poussées latérales de sol. -PLATE-. L.C.P.C. NANTES.

[ 2 ] Coyle, H. and Gisbon, G. C. 1970 . Empirical damping constants for sand and clays. Journal of the Soil Mechanics and Foundations Division, ASCE, Vol. 96, SM, p.946-96.

[ 3 ] Hunt. K. H , Crossley. F. R. E. 1975. Coefficient of restitution interpreted as damping in vibroimpact. Journal of Appliea Miechanics.

[ 4 ] Mastouri, F. 1986. Eiüúe des rideaux de soutènemeni semi-flexibles: Erude théorique et expérimentale. Thèse de Doctorat, I.N.S.A de LYON.

[5] Panovko. J. G. 1977. Introduction à la théorie du choc mécanique. Edition NANKA . MOSCOU.

[ 6] Roger. A, Brown. 1972. Soil parameters required to simulate the dynamic lateral response of model in stiff clay. Fourth Annual Offshore Technology Conference. HOUSTON.

[ 7 ] Ross, H. E., Ir. 1970. Dynamic response of offshore piling. PhD Dissertation. TEXAS - University.

[ 8 ] Samson, C. H., Jr., Hirsch, T. J., and Lowery, L.L. 1963. Computer study of dynamic behavior of piling. Journal of the Structural Division. ASCE. ST4. Paper $N^{\circ} 3608$.

[ 9 ] Smith, E. A. L. 1970. Pile driving analysis by the wave equation. Journal of the Soil Mechanics and Foundation Division. ASCE. Paper $\mathrm{N}^{\circ} 2574$. SM4. 


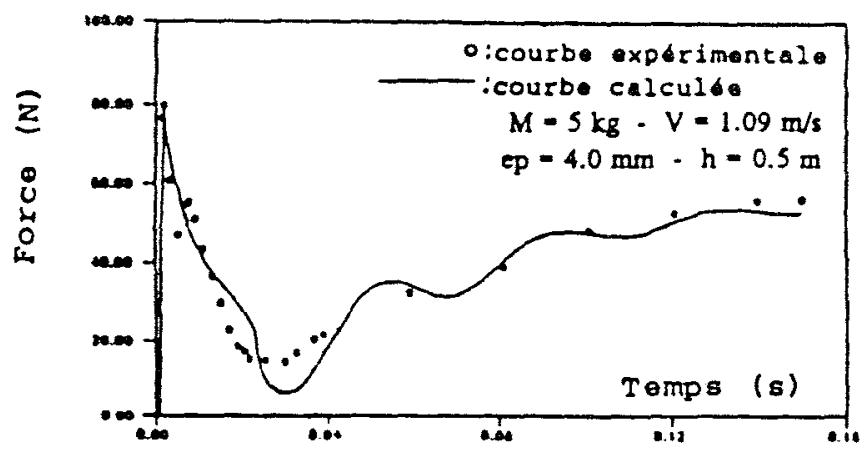

Fig. 7.1 - Force en tête en fonction du temps

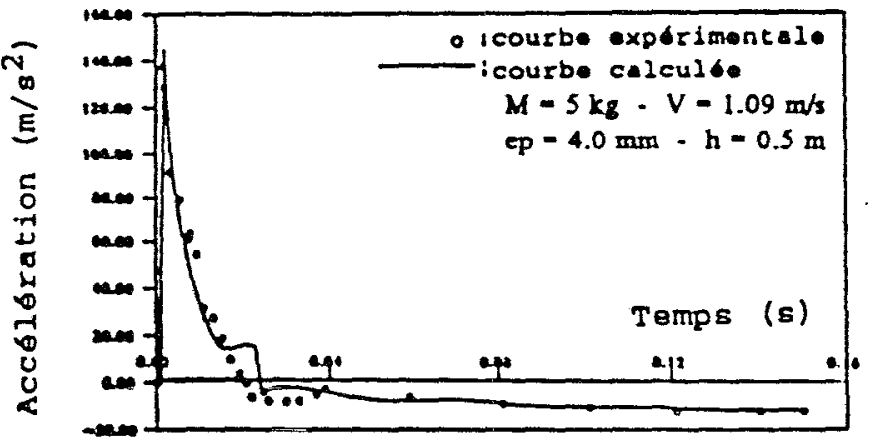

Fig. 7.2 - Accélération en tête en fonction du temps

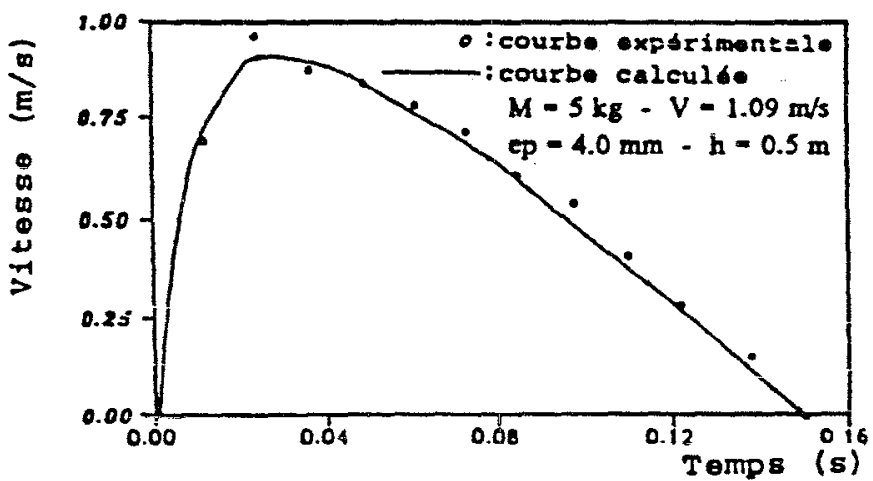

Fig. 7.3 - Vititesše en tête en fonction du temps

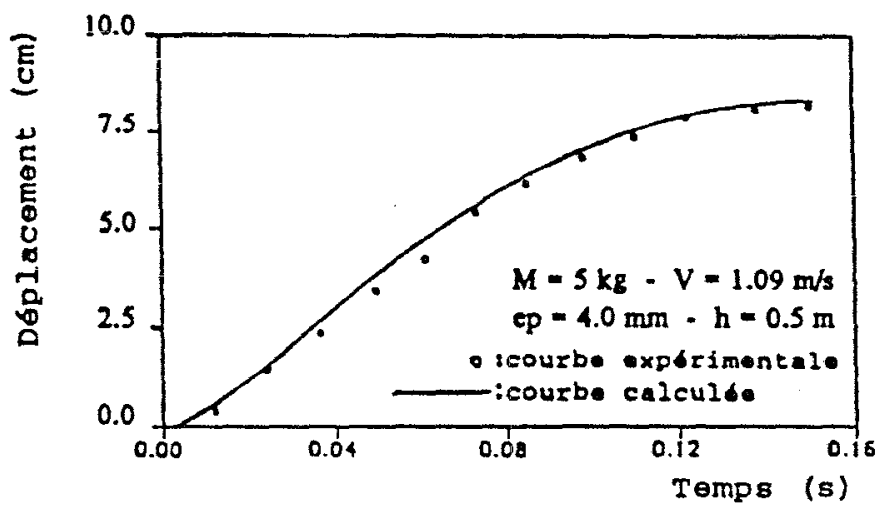

Fig. 7.4 - Déplacement en tête en fonction du temps 


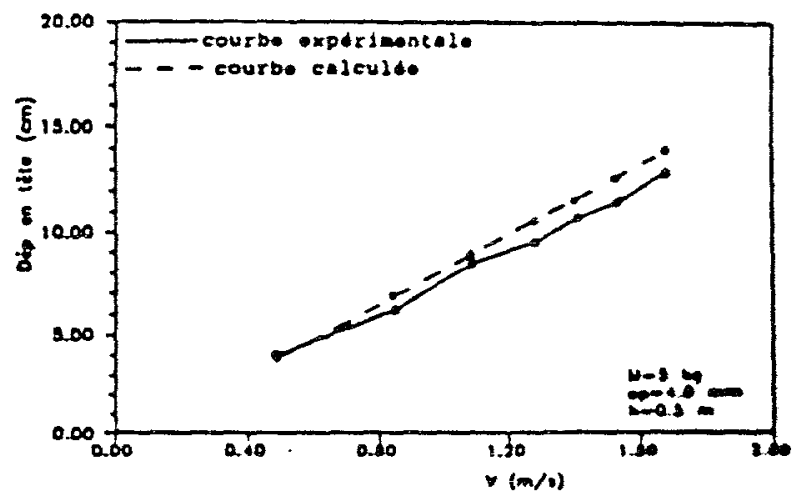

Fig. 8.1 - Déplacement maximal en tête f (vitesse ormpac $\eta$

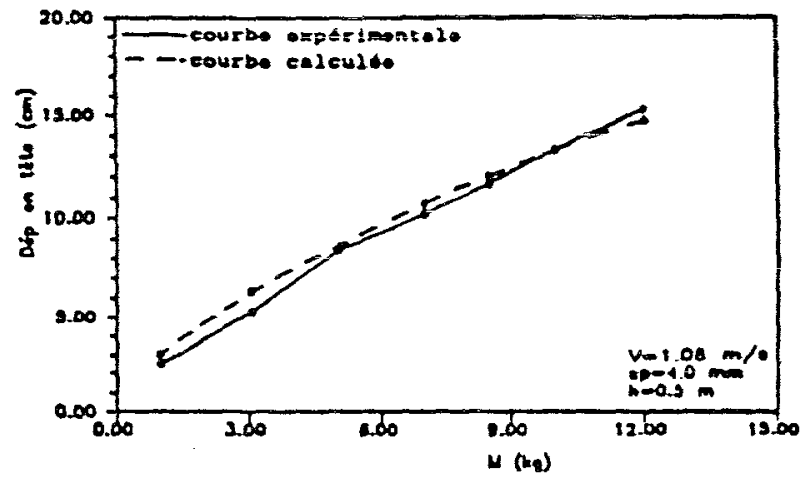

Fig. 8.2 - Déplacement maximal en tête f (masse dimpact M)

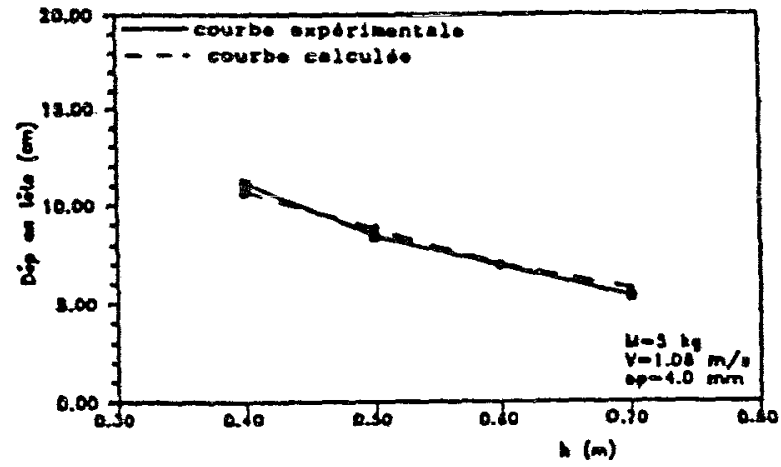

Fig. 8.3 - Déplacement maximal en tête f (fiche $h$ )

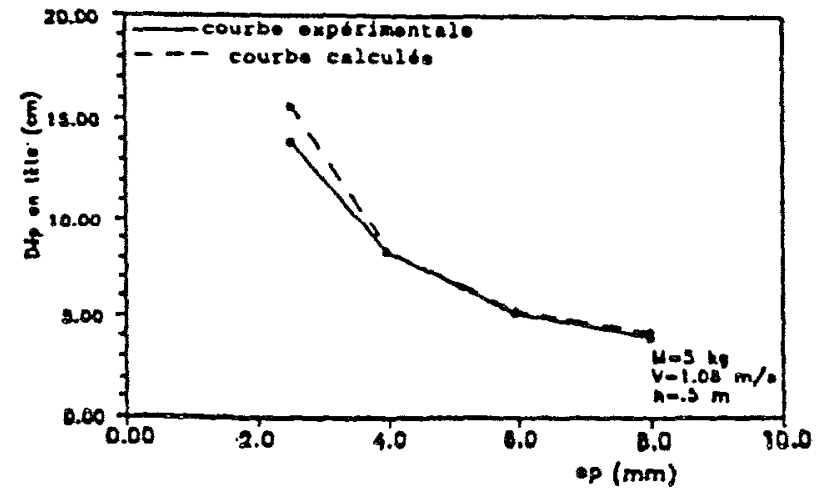

Fig. 8.4 - Déplacement maximal en tête f (épaisseur de la plaque ep) 